\title{
Incidence and risk factors of COVID-19-like symptoms in the French general population during the lockdown period: a multi-cohort study
}

Fabrice Carrat ${ }^{1,2^{*}}$ (D) Mathilde Touvier ${ }^{3}$, Gianluca Severi ${ }^{4,5}$, Laurence Meyer ${ }^{6,7}$, Florence Jusot ${ }^{8}$, Nathanael Lapidus ${ }^{1,2}$, Delphine Rahib ${ }^{9}$, Nathalie Lydié ${ }^{9}$, Marie-Aline Charles $^{10}$, Pierre-Yves Ancel ${ }^{11,12}$, Alexandra Rouquette ${ }^{5,6}$,

Xavier de Lamballerie ${ }^{13}$, Marie Zins ${ }^{14,15}$, Nathalie Bajos ${ }^{16}$ and for the SAPRIS study group

\begin{abstract}
Background: Our main objectives were to estimate the incidence of illnesses presumably caused by SARS-CoV-2 infection during the lockdown period and to identify the associated risk factors.

Methods: Participants from 3 adult cohorts in the general population in France were invited to participate in a survey on COVID-19. The main outcome was COVID-19-Like Symptoms (CLS), defined as a sudden onset of cough, fever, dyspnea, ageusia and/or anosmia, that lasted more than 3 days and occurred during the 17 days before the survey. We used delayed-entry Cox models to identify associated factors.

Results: Between April 2, 2020 and May 12, 2020, 279,478 participants were invited, 116,903 validated the questionnaire and 106,848 were included in the analysis. Three thousand thirty-five cases of CLS were reported during 62,099 person-months of follow-up. The cumulative incidences of CLS were 6.2\% (95\% Confidence Interval (95\%Cl): 5.7\%; 6.6\%) on day 15 and 8.8\% (95\%Cl 8.3\%; 9.2\%) on day 45 of lockdown. The risk of CLS was lower in older age groups and higher in French regions with a high prevalence of SARS-CoV-2 infection, in participants living in cities $>100,000$ inhabitants (vs rural areas), when at least one child or adolescent was living in the same household, in overweight or obese people, and in people with chronic respiratory diseases, anxiety or depression or chronic diseases other than diabetes, cancer, hypertension or cardiovascular diseases.

Conclusion: The incidence of CLS in the general population remained high during the first 2 weeks of lockdown, and decreased significantly thereafter. Modifiable and non-modifiable risk factors were identified.
\end{abstract}

Keywords: COVID-19, General population, Cohort, Incidence, Risk factors, SARS-CoV-2

\footnotetext{
* Correspondence: fabrice.carrat@iplesp.upmc.fr

'Sorbonne Université, Inserm, Institut Pierre-Louis d'Epidémiologie et de Santé Publique, 27 rue Chaligny, 75571 CEDEX 12 Paris, France

2Département de Santé Publique, APHP. Sorbonne Université, Paris, France

Full list of author information is available at the end of the article
}

\section{Strengths and limitations of this study}

- Lockdown was associated with a strong decrease in the incidence of COVID-19-Like Symptoms (CLS) in the French adult population.

- We identified several risk factors of CLS during this period, and we described the immediate

C C The Author(s). 2021 Open Access This article is licensed under a Creative Commons Attribution 4.0 International License, which permits use, sharing, adaptation, distribution and reproduction in any medium or format, as long as you give appropriate credit to the original author(s) and the source, provide a link to the Creative Commons licence, and indicate if changes were made. The images or other third party material in this article are included in the article's Creative Commons licence, unless indicated otherwise in a credit line to the material. If material is not included in the article's Creative Commons licence and your intended use is not permitted by statutory regulation or exceeds the permitted use, you will need to obtain permission directly from the copyright holder. To view a copy of this licence, visit http://creativecommons.org/licenses/by/4.0/ The Creative Commons Public Domain Dedication waiver (http://creativecommons.org/publicdomain/zero/1.0/) applies to the data made available in this article, unless otherwise stated in a credit line to the data. 
consequences in terms of access to healthcare and treatment.

- The most important limitation was the lack of virological confirmation of CLS and the risk of misclassification of a SARS-CoV-2 infection and a disease from another etiology.

- Although participation bias was accounted for with an appropriate weighting method, our findings should not be considered to be strictly representative of the general adult population in France

\section{Introduction}

Following the identification of a novel coronavirus (SARSCoV-2) in Wuhan, China in December 2019 and its worldwide spread [1], the first imported COVID-19 cases were initially reported in France on January 24, 2020 [2]. Less than 2 months later, the French government declared a nationwide epidemic (phase 3) and a generalized lockdown procedure was set-up on March 17, 2020 [3]. The lockdown included banning of any non-essential public gatherings, closure of educational and public/cultural institutions, ordering people to stay home apart from exercise and essential tasks. Children and their parents were required to stay at home as much as possible [4]. Public health reports have shown that lockdown had a marked impact on the dynamics of the pandemic with a clear downward trend in new hospitalizations from April 1, 2020 , and a consecutive decrease in the number of deaths from April 7, 2020 [4, 5]. Thus, the French government eased these restrictions on May 11, 2020 [3]. Although lockdown appeared to successfully alleviate the burden of severe COVID-19 [6], estimates of its impact on mild-tomoderate COVID-19 are based on modelling studies [7], and are not yet supported by clinical evidence.

Our main goals were 1) to estimate the incidence of illnesses presumably caused by SARS-CoV-2 infection during the lockdown period; 2) to identify the associated risk factors. We also described associated symptoms, preventive behaviors and healthcare in relation to these illnesses.

\section{Participants and methods \\ Design}

The SAPRIS ("SAnté, Perception, pratiques, Relations et Inégalités Sociales en population générale pendant la crise COVID-19") survey was began in March 2020 to evaluate the main epidemiological, social and behavioral challenges of the SARS-CoV2 epidemic in France in relation to social inequalities in health and healthcare. SAPRIS is based on a consortia of prospective cohort studies involving two child-cohorts (not presented in this study) and three general population-based adult cohorts:

- 1) CONSTANCES, a "general population" cohort including 204,973 adults aged 18 to 69 at inclusion and randomly selected from 2012 to be a representative sample of the French adult population affiliated to the General Health Insurance Fund (the source population, that is, approximately $85 \%$ of the total French population) [8]. Among CONSTANCES participants, 66,881 are followed by internet, the rest through mailed questionnaires.

- 2) E3N / E4N, a multigenerational adult cohort based on a community of families with 113,000 participants (including women recruited in 1990 and still actively followed-up, their offspring and the fathers of these offspring) among whom 89,606 followed by internet, the rest through mailed questionnaires [9].

- 3) NutriNet-Santé, a nutritional general populationbased internet cohort started in 2009, with 170,000 included participants among whom 151,122 were still followed-up in 2020 [10].

Ethics and public involvement.

Ethical approval and written informed consent was obtained from each participant before enrolment in the original cohort. According to French law, the present nested survey did not require specific additional written consent from the participant. It was approved by the Inserm ethics evaluation committee (approval \#20-672 dated March 30, 2020). Volunteer participants were involved in testing the readability, the comprehension and acceptability of the questions as well as the time required to complete the questionnaires, but they did not contribute to other aspects related to the design, conduct, reporting or dissemination of the research.

All participants from the original cohorts followed using electronic (internet) questionnaires and who were still under active follow-up on April 1, $2020(n=279,478)$ were invited to participate in the current SAPRIS survey (Fig. 1). There were no restrictions on inclusion criteria in the survey. A first self-administered questionnaire covered the lockdown period and was sent from April 1, 2020 and returned before May 12, 2020. A second questionnaire covered the postlockdown period and was sent between May 5, 2020 and June 15, 2020. The present study used the data from the first self-administered questionnaire, which included questions on socio-demographics, household size and composition, SARS-CoV2 diagnosis, a detailed description of the subject's symptoms in the 2 weeks before the questionnaire, comorbidities, healthcare use and treatment, employment, daily life, child care, alcohol, tobacco and cannabis use, social and sexual life, preventive measures, risk perception and beliefs.

Additional specific socio-demographic and clinical characteristics were extracted from original cohort databases. Symptoms were reported if they had been present at least once in the last 14 days. If a symptom had been, but was no longer present when the questionnaire was completed, the duration was noted on a scale (less than 1 day, one to 3 days, four to 7 days, eight to 14 days, $>14$ days). Finally, the total time (in days) between the onset of the first symptoms and the questionnaire was reported. All visits 


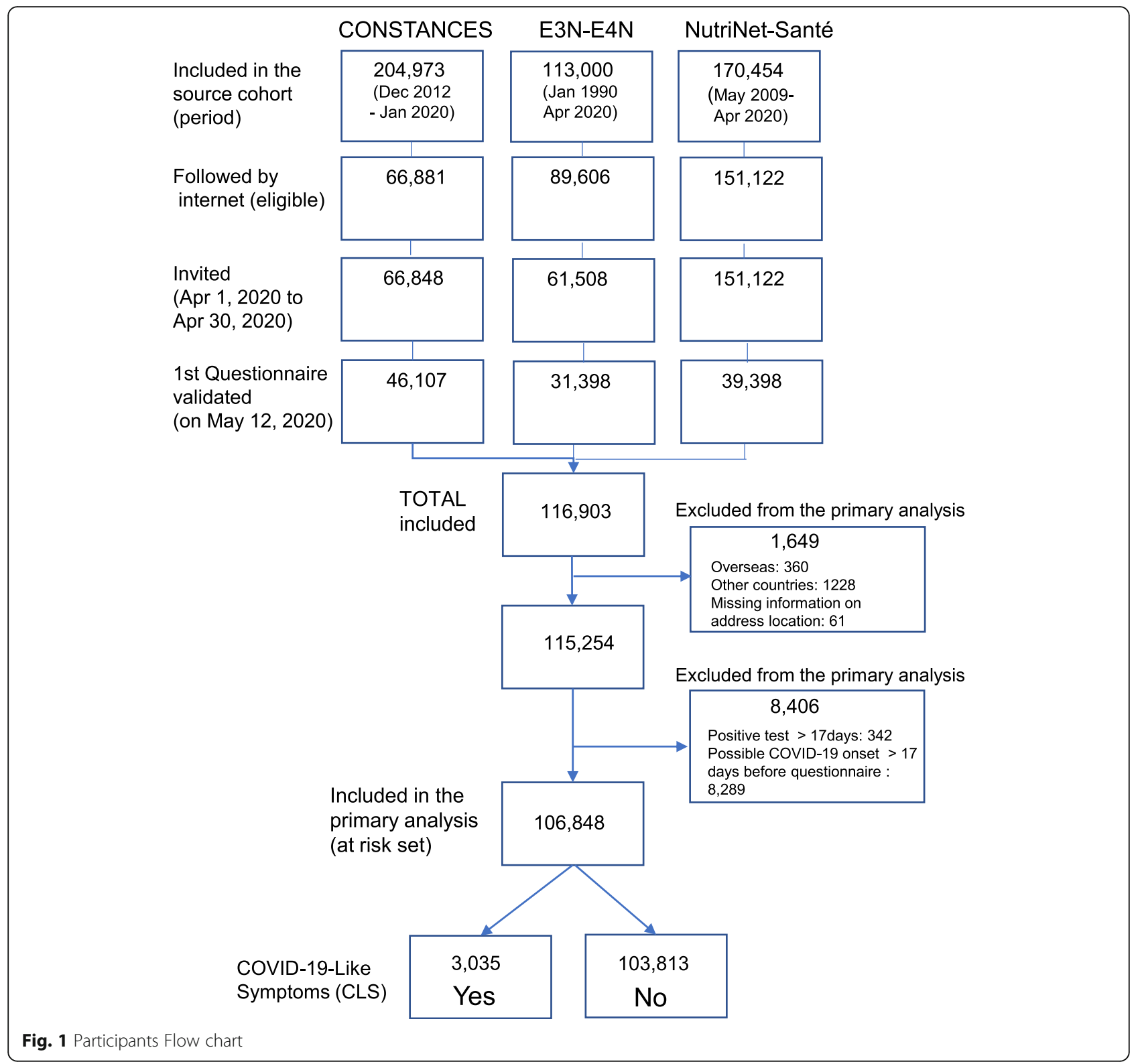

outside the home and the use of preventive measures in the 7 days before the questionnaire were reported.

\section{Outcome}

The main outcome was COVID-19-Like symptoms (CLS), defined according to the European Centre for Disease Prevention and Control as at least one of a cough, a fever, a dyspnea, a sudden onset of anosmia, ageusia or dysgeusia [11], that lasted more than 3 days and occurred during the at-risk period. Participants were also requested to report the occurrence of cough, fever or dyspnea before March 1, 2020 or between March 1 and the 2 weeks before the questionnaire, and whether they or any other household members had tested positive for SARS-CoV-2 before the questionnaire. The primary "at-risk period" was defined as the 17 days before the self- administered questionnaire for each patient, corresponding to the 14 days to report the presence of symptoms, plus 3 days for the minimum duration of our definition of CLS. In a first sensitivity analysis, no restriction was made on the minimum duration of symptoms, extending our primary case-definition of CLS to illness that lasted less than 4 days. In a second sensitivity analysis, the at-risk period was defined as between March 16, 2020 and the date of the questionnaire for all participants. This definition made it possible to include all CLS that occurred during the lockdown period.

\section{Covariates}

We explored the association of CLS with age, gender, address location, French metropolitan regions, number 
of people living in the household, number of children in the household, educational level, professional activity before lockdown, job position, professional activity during lockdown, BMI, chronic conditions (according to a prespecified list). Age groups were categorized according to predefined limits (<40 (reference level); 40-49; 50-59; $60-69$; $\geq 70$ years) and BMI according to standard cutoffs $(<18.5$; 18.5- $<25$ (reference level); $\geq 25$ - $<30$ (overweight); $\geq 30$ (obese) $\mathrm{kg} / \mathrm{m}^{2}$ ) [12].

\section{Statistical methods}

We determined that 100,000 subjects were needed to have a power of at least $80 \%$ to identify associations (Odds-Ratio, OR $<0.9$ or OR $>1.1$ ) between covariates and CLS in a wide range of situations, assuming $10 \%$ of events and 10 to $90 \%$ exposure.

We used inverse probability weighting to correct for selection bias (when only a subgroup of the whole cohort was invited to participate by internet) and inverse probability weighting to correct for non-participation bias in those who were invited. Weights were estimated using logistic regression models, with selection or participation as the response variables, and participants' characteristics as covariates (see supplementary Table 1). Unweighted and weighted daily incidence rates of CLS and $95 \%$ confidence intervals were estimated with an exact method based on the Poisson distribution. Estimates of unweighted and weighted cumulative incidences on days 15 (March 30, 2020), 30 (April 14, 2020) and 45 (April 29, 2020) of lockdown were obtained as one minus the estimated probability of survival free of CLS at that time.

To account for potential heterogeneity between the cohorts, left-truncation and censorship in the data, factors associated with the occurrence of CLS were identified using unweighted data and delayed-entry Cox models with stratification on the source cohort. The start of the at-risk period was defined according to the calendar day for each participant and survival time was calculated as the time between that day and the day the questionnaire was filled-out in case of no symptom or the day the first symptoms were reported in CLS cases. Multivariable analysis was performed including all factors associated with CLS cases on univariable analysis. All analyses were performed with SAS 9.4 software (SAS Institute Inc., Cary, North Carolina, USA). A $P$-value $<.05$ was considered to be statistically significant.

\section{Results}

A total of 116,903 of the 279,478 participants (42\%) who were invited to participate in the survey completed the questionnaire. The participation rate was $69 \%$ in the CONSTANCES cohort, 51\% in the E3NE4N cohorts and $26 \%$ in the NutriNet-Santé cohort (Fig. 1).
Table 1 presents the characteristics of included participants. Median age was 59 years old (Q1-Q3: 46 to 71 years), and $66 \%$ of the participants were women. Twenty-six percent were residents of the Ile-de-France or GrandEst regions - the two regions with the highest rate of SARS-CoV-2 in metropolitan France, while 23\% lived in rural areas and 44\% lived in cities of more than 100,000 inhabitants. At least one child or adolescent was present at home in $25 \%$. Forty-three percent were retired and 50\% were working adults, but only $8 \%$ worked outside the home during the lockdown period. Ten percent of the participants were obese and a chronic disease was reported in $34 \%$ of participants.

Participants who were living outside mainland France ( $n=1588)$ or with missing information on their exact address location $(n=61)$ or who reported a positive SARSCoV-2 test result $(n=342)$ and/or a CLS onset $(n=8289)$ before the at-risk period were excluded from the primary analysis. The primary analysis evaluated 106,848 participants: 3035 CLS were reported during 62,099 personmonths of follow-up. The unweighted cumulative incidences of CLS were $6.2 \%$ (95\% Confidence Interval (95\%CI): $5.7 \%$; $6.6 \%), 7.7 \%$ (95\%CI 7.3\%; $8.2 \%)$ and $8.8 \%$ (95\%CI $8.3 \%$; 9.2\%) on days 15,30 and 45 of lockdown, respectively. The weighted cumulative incidences were $7.2 \%$ (95\%CI 6.7\%; 7.8\%), 9.0\% (95\%CI 8.4\%; 9.5\%) and $10.1 \%$ $(95 \% \mathrm{CI} 9.6 \% ; 10.6 \%)$ on days 15,30 and 45 of lockdown, respectively.

Extending CLS definition to illness that lasted less than 4 days, 5313 cases were identified during 59,768 personmonths of follow-up with unweighted cumulative incidences of 9.7\% (95\%CI 9.2\%; 10.3\%), 12.6\% (95\%CI 12.0\%; $13.1 \%$ ) and $14.3 \%$ (95\%CI 13.7\%;14.9\%) on days 15,30 and 45 of lockdown, respectively. Sensitivity analysis of all cases of CLS onset after March 16, 2020 excluded 171 participants with a positive test result and/or 4084 with the onset of CLS before March 16, 2020 and identified 7240 cases in 110,207 person-months of follow-up with unweighted cumulative incidences of $4.7 \%$ (95\%CI $4.5 \%$; $4.8 \%$ ), $6.6 \%$ (95\% CI 6.5\%; 6.8\%) and 7.7\% (95\% CI 7.5\%; $7.9 \%$ ) on days 15,30 and 45 of lockdown, respectively.

The primary daily incidence rate peaked on day four of lockdown (March 19, 2020; unweighted estimate 5.57 per 1000 person-days (95\%CI 4.45; 6.89) - Fig. 2) and showed a sharp and constant decrease to reach less than 1 per 1000 person-days after day 25 (April 9, 2020). Similar findings were observed in the weighted incidence rates and the sensitivity analysis considering a different at-risk period (supplementary Figs. 1\&2). Daily incidence rates were higher but showed a similar temporal pattern when the case-definition of CLS included illness that lasted less than 4 days (supplementary Fig. 3).

Eighty out of 189 participants who experienced CLS and were tested reported a positive (RT-PCR) test result (supplementary Table 2). Headaches, rhinorrhea and 
Table 1 Participants' characteristics

\begin{tabular}{|c|c|c|c|c|c|c|}
\hline & \multirow[t]{2}{*}{$\begin{array}{l}\text { Constances } \\
N=46,107(39)^{\mathrm{a}}\end{array}$} & \multicolumn{3}{|c|}{$\begin{array}{l}\text { E3NE4N } \\
N=31,398\end{array}$} & \multirow[t]{2}{*}{$\begin{array}{l}\text { NutriNet-Santé } \\
N=39,398 \text { (34) }\end{array}$} & \multirow[t]{2}{*}{$\begin{array}{l}\text { Total adults } \\
116,903(100)\end{array}$} \\
\hline & & $\begin{array}{l}\text { E3N } \\
N=16,744\end{array}$ & $\begin{array}{l}\text { E4NG1 } \\
N=4865\end{array}$ & $\begin{array}{l}\text { E4NG2 } \\
N=9789\end{array}$ & & \\
\hline \multicolumn{7}{|l|}{ Questionnaires } \\
\hline First & Apr 6, 2020 & Apr 17, 2020 & Apr 17, 2020 & Apr 9, 2020 & Apr 1, 2020 & Apr 1, 2020 \\
\hline Last & May 5, 2020 & May 8, 2020 & May 7, 2020 & May 6, 2020 & May 12, 2020 & May 12, 2020 \\
\hline \multicolumn{7}{|l|}{ Age group (years) } \\
\hline$<40$ & $9504(21)^{\mathrm{a}}$ & $0(0)$ & $0(0)$ & $764(8)$ & $6296(16)$ & $16,564(14)$ \\
\hline$[40-50[$ & $10,483(23)$ & $0(0)$ & $0(0)$ & $4016(41)$ & $6701(17)$ & $21,200(18)$ \\
\hline$[50-60[$ & $9400(20)$ & $0(0)$ & $3(1)$ & 3820 (39) & 7799 (20) & $21,022(18)$ \\
\hline$[60-70[$ & $11,408(25)$ & $0(0)$ & $273(6)$ & $1146(12)$ & $9933(25)$ & $22,760(19)$ \\
\hline$>=70$ & $5312(16)$ & $16,744(100)$ & 4589 (94) & $43(0)$ & 8669 (22) & $35,357(30)$ \\
\hline \multicolumn{7}{|l|}{ Gender } \\
\hline Female & $23,426(51)$ & $16,744(100)$ & $0(0)$ & $6483(66)$ & $30,130(76)$ & $76,783(66)$ \\
\hline Male & $22,681(49)$ & $0(0)$ & $4865(100)$ & 3306 (34) & 9268 (34) & $40,120(34)$ \\
\hline \multicolumn{7}{|l|}{ Regions } \\
\hline Ile-de-France & 7856 (17) & $2760(16)$ & $707(15)$ & $2440(25)$ & 7280 (18) & $21,043(18)$ \\
\hline Grand-Est & $4165(9)$ & $1308(8)$ & $387(8)$ & $615(6)$ & $2903(7)$ & $9378(8)$ \\
\hline Other French metropolitan regions & $34,086(74)$ & $12,673(76)$ & $3770(77)$ & $6218(64)$ & $28,086(71)$ & $84,833(73)$ \\
\hline French Overseas & $0(0)$ & $0(0)$ & $1(0)$ & $54(1)$ & $305(1)$ & $360(0)$ \\
\hline Foreign countries & $0(0)$ & $3(0)$ & $0(0)$ & $401(4)$ & $824(2)$ & $1228(1)$ \\
\hline Missing & 0 & 0 & 0 & 61 & 0 & 61 \\
\hline \multicolumn{7}{|l|}{ Living Area } \\
\hline Rural & $3349(8)$ & $5125(31)$ & $1613(33)$ & $2784(29)$ & $13,550(34)$ & $26,421(23)$ \\
\hline$<20,000$ inhab. & $1310(3)$ & $4526(27)$ & $1454(30)$ & $2330(24)$ & $9174(23)$ & $18,794(16)$ \\
\hline 20-000-100,000 inhab. & $2871(7)$ & $3989(24)$ & $1061(22)$ & $2254(23)$ & $8886(23)$ & $19,061(17)$ \\
\hline$>100,000$ inhab. & $36,473(83)$ & 3005 (18) & $722(15)$ & $2389(24)$ & $7788(20)$ & $50,377(44)$ \\
\hline Missing & 2104 & 99 & 15 & 32 & 0 & 2250 \\
\hline \multicolumn{7}{|l|}{ Household size and composition } \\
\hline \multicolumn{7}{|l|}{$\mathrm{Nb}$ persons (incl. participant) } \\
\hline 1 & $6461(14)$ & $4869(30)$ & $168(4)$ & $1200(12)$ & $7855(20)$ & $20,553(18)$ \\
\hline 2 & $18,963(41)$ & $10,601(65)$ & $4260(91)$ & $2465(25)$ & $18,032(46)$ & $54,231(47)$ \\
\hline $3-6$ & $20,274(44)$ & $751(5)$ & $253(5)$ & $5990(61)$ & $13,360(34)$ & $40,268(35)$ \\
\hline 7 or + & $248(1)$ & $44(0)$ & $6(0)$ & $87(1)$ & $151(0)$ & $536(0)$ \\
\hline Missing & 161 & 479 & 178 & 47 & 0 & 865 \\
\hline \multicolumn{7}{|l|}{$\mathrm{Nb}$ children $(<18 \mathrm{yrs})$} \\
\hline 0 & $31,462(68)$ & $16,089(99)$ & 4640 (99) & $5033(52)$ & $29,841(76)$ & $87,908(75)$ \\
\hline 1 & $5637(12)$ & $100(1)$ & $33(1)$ & $1852(19)$ & $4063(10)$ & $11,693(10)$ \\
\hline 2 & $6761(15)$ & $61(0)$ & $11(0)$ & $2151(22)$ & $4189(11)$ & $13,186(11)$ \\
\hline $3-6$ & $2078(5)$ & $13(0)$ & $3(0)$ & $701(7)$ & $1301(3)$ & $4097(4)$ \\
\hline 7 or + & $8(0)$ & $2(0)$ & $0(0)$ & $5(0)$ & $4(0)$ & $19(0)$ \\
\hline Missing & 161 & 479 & 178 & 47 & 0 & 865 \\
\hline \multicolumn{7}{|l|}{ Educational level } \\
\hline$<$ High-school degree & $5843(13)$ & $971(6)$ & $595(12)$ & & $5830(15)$ & $13,239(13)$ \\
\hline High-school degree or undergraduate & $23,839(53)$ & $7486(46)$ & $1335(28)$ & & $17,247(44)$ & $49,907(47)$ \\
\hline Graduate degree or doctorate & $15,257(34)$ & $7790(48)$ & $2906(60)$ & & $16,060(41)$ & $42,013(40)$ \\
\hline Other or Missing & 1168 & 497 & 29 & 9789 & 261 & 11,744 \\
\hline
\end{tabular}


Table 1 Participants' characteristics (Continued)

\begin{tabular}{|c|c|c|c|c|c|c|}
\hline & \multirow[t]{2}{*}{$\begin{array}{l}\text { Constances } \\
N=46,107(39)^{\mathrm{a}}\end{array}$} & \multicolumn{3}{|c|}{$\begin{array}{l}\text { E3NE4N } \\
N=31,398\end{array}$} & \multirow[t]{2}{*}{$\begin{array}{l}\text { NutriNet-Santé } \\
N=39,398 \text { (34) }\end{array}$} & \multirow[t]{2}{*}{$\begin{array}{l}\text { Total adults } \\
116,903(100)\end{array}$} \\
\hline & & $\begin{array}{l}\mathrm{E3N} \\
N=16,744\end{array}$ & $\begin{array}{l}\text { E4NG1 } \\
N=4865\end{array}$ & $\begin{array}{l}\text { E4NG2 } \\
N=9789\end{array}$ & & \\
\hline \multicolumn{7}{|l|}{ Professional activity before lockdown } \\
\hline Student & $402(1)$ & $6(0)$ & $0(0)$ & $19(0)$ & $426(1)$ & $853(1)$ \\
\hline Working & $29,153(63)$ & $47(0)$ & $31(1)$ & $8299(85)$ & $20,529(52)$ & $58,059(50)$ \\
\hline Looking for a job & $1543(3)$ & $1(0)$ & $3(0)$ & $336(3)$ & $1128(3)$ & $3111(3)$ \\
\hline Retired & $13,678(30)$ & $16,188(97)$ & $4705(97)$ & $756(8)$ & $15,368(39)$ & $50,695(43)$ \\
\hline Not working due to health conditions & $383(1)$ & $9(0)$ & $3(0)$ & $110(1)$ & $552(1)$ & $1057(1)$ \\
\hline No professional activity (housewife or husband) & $806(2)$ & $438(3)$ & $113(2)$ & $205(2)$ & $1295(3)$ & $2857(2)$ \\
\hline Missing & 142 & 55 & 10 & 64 & 0 & 271 \\
\hline \multicolumn{7}{|l|}{ Essential job position } \\
\hline Healthcare worker & $1968(4)$ & $0(0)$ & $1(0)$ & $555(6)$ & $1744(4)$ & $4268(4)$ \\
\hline Other essential job & $5330(12)$ & $6(0)$ & $2(0)$ & $1423(15)$ & $4250(11)$ & $11,011(9)$ \\
\hline \multicolumn{7}{|l|}{ Professional activity during lockdown } \\
\hline Not working & $16,812(36)$ & $16,642(100)$ & $4824(100)$ & $1426(16)$ & $18,869(53)$ & $58,873(54)$ \\
\hline Stopped working & $2304(5)$ & & $0(0)$ & $423(5)$ & $1703(5)$ & $4442(4)$ \\
\hline Working from home, remote working & $15,030(35)$ & $12(0)$ & $14(0)$ & $5015(56)$ & $8910(25)$ & $28,986(27)$ \\
\hline Partially working from home & $2908(7)$ & $17(0)$ & $5(0)$ & $899(10)$ & $2201(6)$ & $6015(6)$ \\
\hline Working outside home & $4189(10)$ & $2(0)$ & $1(0)$ & $902(10)$ & $3614(10)$ & 8707 (8) \\
\hline Other & $1321(3)$ & $1(0)$ & $3(0)$ & $359(4)$ & $104(0)$ & $1789(2)$ \\
\hline Missing & 3543 & $\begin{array}{l}2(0) \\
68\end{array}$ & 18 & 765 & 3997 & 8391 \\
\hline \multicolumn{7}{|l|}{ BMI $\left(\mathrm{kg} / \mathrm{m}^{2}\right)$} \\
\hline$<18.5$ & $1147(2)$ & $633(4)$ & $31(1)$ & $296(4)$ & $1752(5)$ & 3859 (3) \\
\hline$[18.5 ; 25[$ & $26,254(58)$ & $9621(59)$ & $2385(49)$ & $5173(63)$ & $23,054(60)$ & $66,487(59)$ \\
\hline [25; 30[(overweight) & $13,320(30)$ & $4597(28)$ & $2023(42)$ & $1944(24)$ & $9538(25)$ & $31,422(28)$ \\
\hline$>=30$ (obese) & $4173(9)$ & $1402(9)$ & $382(8)$ & $763(9)$ & $4098(11)$ & $10,818(10)$ \\
\hline Missing & 1213 & 491 & 44 & 1613 & 956 & 3873 \\
\hline \multicolumn{7}{|l|}{ Chronic diseases } \\
\hline Yes & $11,777(26)$ & $7984(48)$ & $2826(58)$ & $2338(24)$ & $14,310(36)$ & 39,235 (34) \\
\hline No & $33,891(74)$ & $8490(51)$ & $1977(41)$ & $7378(75)$ & $24,752(21)$ & $76,488(66)$ \\
\hline Don't know & $284(1)$ & $195(1)$ & $51(1)$ & $65(1)$ & $336(1)$ & $931(1)$ \\
\hline Missing & 155 & 75 & 11 & 8 & 0 & 249 \\
\hline \multicolumn{7}{|l|}{ Chronic diseases } \\
\hline Asthma, COPD, other respir. Diseases & 1399 (3) & $5794(35)$ & $1572(32)$ & $295(3)$ & $7042(18)$ & $16,102(14)$ \\
\hline Diabetes & $690(2)$ & $3252(20)$ & $1127(23)$ & $127(1)$ & $1417(4)$ & $6613(6)$ \\
\hline Hypertension & $2993(7)$ & $3275(20)$ & $1218(25)$ & $469(5)$ & $4787(12)$ & $12,742(11)$ \\
\hline Other cardiovascular diseases & $934(2)$ & $1012(6)$ & $727(15)$ & $101(1)$ & $1293(3)$ & 4067 (3) \\
\hline Cancer & $602(1)$ & $755(5)$ & $426(9)$ & $108(1)$ & $4525(11)$ & $6416(6)$ \\
\hline Anxiety, depression & $1083(2)$ & $619(4)$ & $106(2)$ & $267(3)$ & $1323(3)$ & $3398(3)$ \\
\hline Other & $3253(7)$ & $3430(21)$ & $791(16)$ & $569(6)$ & 7408 (19) & $15,451(13)$ \\
\hline Missing & 142 & 55 & 10 & 64 & 0 & 249 \\
\hline
\end{tabular}

${ }^{\mathrm{a}} \mathrm{n}(\%)$ 


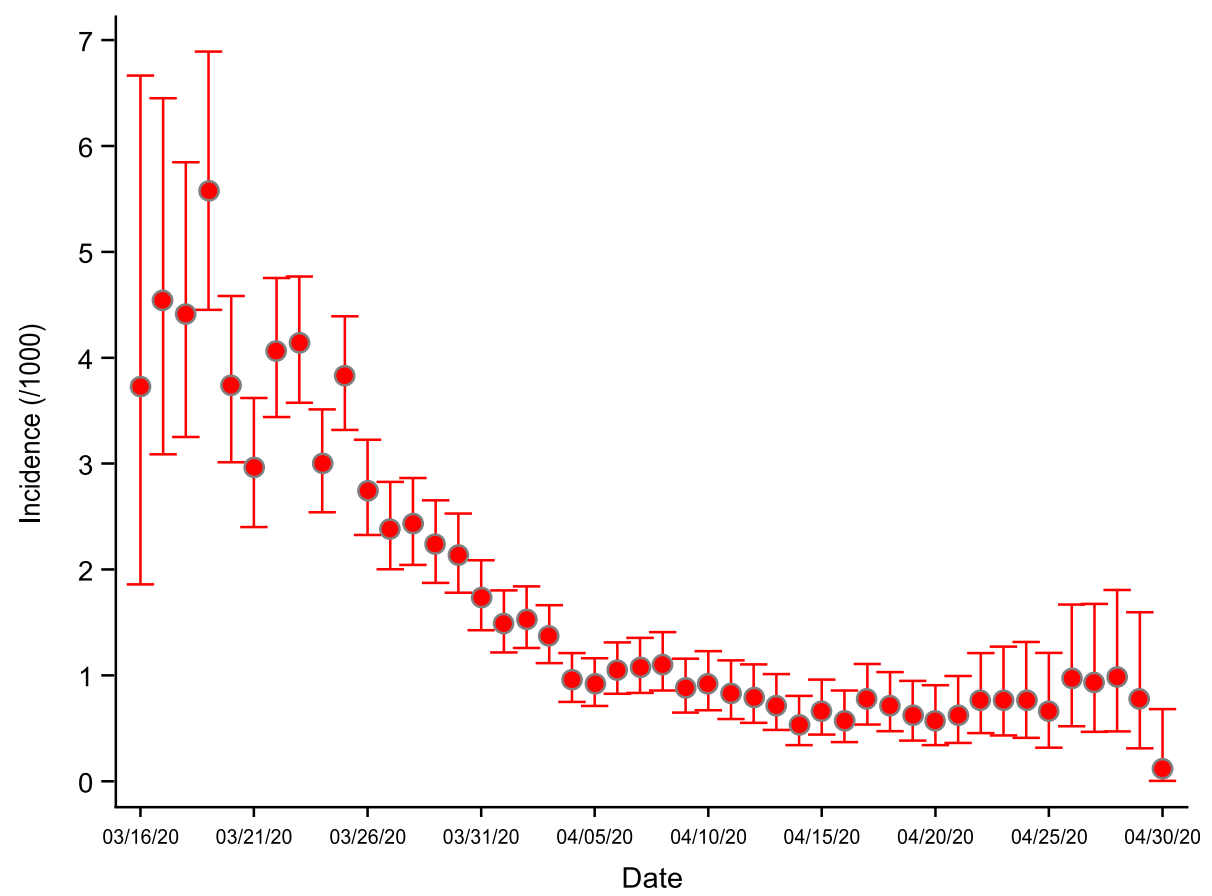

Fig. 2 Daily incidence (/1000) of COVID-19-Like Symptoms (> 3 days) in participants between March 16, 2020 (first day of the lockdown in France) and April 30, 2020 - unweighted estimates. Error bars indicate 95\% confidence intervals

fatigue were frequently reported in addition to the symptoms defining CLS. Eight hundred and forty-eight (28\%) participants with CLS had a GP or a hospital visit, and a diagnosis of COVID-19 was considered to be very likely or likely by the physician in $62 \%$ cases. Paracetamol was taken by $62 \%$ and antibiotics by $6 \%$ of participants with CLS. Only 8 participants used chloroquine or hydroxychloroquine. Forty percent participants stayed strictly confined at home following symptoms onset.

Table 2 presents the unweighted incidence rates of CLS and the hazard ratios obtained from the univariable Cox models with stratification on source cohort. Almost all tested factors were found to be associated with CLS. A positive RT-PCR in another household member was strongly associated with CLS in the participant; this variable was not included in the multivariable analysis to avoid overfitting. On multivariable analysis (Table 3), the risk of COVID-19 was lower in older age groups and was higher in the Ile-de-France and GrandEst regions (compared to other French metropolitan regions), in those living in cities $>100,000$ inhabitants (vs rural areas), when at least one child or adolescent was living in the same household, in overweight or obese participants, and in people with chronic respiratory diseases, anxiety or depression and chronic diseases other than diabetes, cancer, hypertension or other cardiovascular diseases. The observed associations were confirmed in the sensitivity analyses, except that male gender, living in a household of size 2 and being retired were negatively associated with the risk of CLS in addition to factors identified in the primary analysis (supplementary Tables 3 \& 4).

\section{Discussion}

Lockdown was associated with a strong decrease in the incidence of CLS in the French adult population that participated in this survey. This study shows that the cumulative incidence of CLS on day 45 of lockdown ranged from 7.7 to $10.2 \%$ depending on the estimation method, that more than $60 \%$ of new cases occurred within the first 2 weeks, and that the daily incidence remained at a sustained low level 1 month after lockdown and thereafter. In addition, we identified several risk factors of CLS during this period, and have described the immediate consequences in terms of access to healthcare and treatment associated with these syndromes. To our knowledge this is the first study to report the signs and symptoms of COVID-19 on a nationwide scale and during lockdown.

Only $28 \%$ of the participants with CLS had a medical visit. This result is in line with estimates based on a digital participatory system in France during the same period, in which $31 \%$ of COVID-19 patients sought medical advice [13]. Forty percent of the participants with symptoms remained strictly confined without leaving their homes, following the government's 
Table 2 Unweighted incidence rates of COVID-19-Like Symptoms (CLS) by covariate values and univariable hazard-ratios ${ }^{a}$

\begin{tabular}{|c|c|c|c|c|}
\hline & $\begin{array}{l}\text { Number CLS } \\
\text { /number person-months }\end{array}$ & $\begin{array}{l}\text { Incidence rate }(95 \% \mathrm{Cl}) \\
\text { (/100 person-months) }\end{array}$ & Hazard-Ratio & $P$-Value \\
\hline \multicolumn{5}{|l|}{ Age group (years) } \\
\hline$<40$ & $732 / 8561$ & $8.55(7.94 ; 9.19)$ & Reference & \\
\hline$[40-50[$ & $783 / 10,980$ & $7.13(6.64 ; 7.65)$ & $0.87(0.78 ; 0.96)$ & 0.0053 \\
\hline$[50-60[$ & $619 / 10,912$ & $5.67(5.23 ; 6.14)$ & $0.68(0.61 ; 0.75)$ & $<0.0001$ \\
\hline$[60-70[$ & $480 / 12,120$ & $3.96(3.61 ; 4.33)$ & $0.43(0.38 ; 0.48)$ & $<0.0001$ \\
\hline$>=70$ & $421 / 19,526$ & $2.16(1.96 ; 2.37)$ & $0.27(0.23 ; 0.31)$ & $<0.0001$ \\
\hline \multicolumn{5}{|l|}{ Gender } \\
\hline Female & $1937 / 40,626$ & $4.77(4.56 ; 4.99)$ & reference & \\
\hline Male & $1098 / 21,473$ & $5.11(4.82 ; 5.43)$ & $0.88(0.81 ; 0.95)$ & 0.0011 \\
\hline \multicolumn{5}{|l|}{ Regions } \\
\hline Ile-de-France & $697 / 11,081$ & $6.29(5.83 ; 6.78)$ & $1.38(1.27 ; 1.51)$ & $<0.0001$ \\
\hline GrandEst & $286 / 4908$ & $5.83(5.17 ; 6.54)$ & $1.30(1.15 ; 1.48)$ & $<0.0001$ \\
\hline Other French metropolitan regions & $2052 / 46,110$ & $4.45(4.26 ; 4.65)$ & reference & \\
\hline \multicolumn{5}{|l|}{ Living Area } \\
\hline Rural & $539 / 14,249$ & $3.78(3.47 ; 4.12)$ & Reference & \\
\hline$<20,000$ inhab. & $386 / 10,069$ & $3.83(3.46 ; 4.24)$ & $1.07(0.94 ; 1.22)$ & 0.2903 \\
\hline $20-000-100,000$ & $465 / 10,114$ & $4.60(4.19 ; 5.04)$ & $1.23(1.09 ; 1.40)$ & 0.0010 \\
\hline$>100,000$ inhab. & $1581 / 26,458$ & $5.98(5.68 ; 6.28)$ & $1.26(1.12 ; 1.41)$ & $<0.0001$ \\
\hline Missing & $64 / 1209$ & $5.29(4.08 ; 6.76)$ & & \\
\hline \multicolumn{5}{|l|}{ Household size and composition } \\
\hline \multicolumn{5}{|l|}{ Nb persons (incl. participant) } \\
\hline 1 & $495 / 10,881$ & $4.55(4.16 ; 4.97)$ & Reference & \\
\hline 2 & $1076 / 29,241$ & $3.68(3.46 ; 3.91)$ & $0.81(0.73 ; 0.91)$ & 0.0002 \\
\hline 3 or + & $1453 / 21,496$ & $6.76(6.42 ; 7.12)$ & $1.36(1.23 ; 1.51)$ & $<0.0001$ \\
\hline Missing & $11 / 481$ & $2.29(1.14 ; 4.09)$ & & \\
\hline \multicolumn{5}{|l|}{$\mathrm{Nb}$ children (< $18 \mathrm{yrs})$} \\
\hline 0 & $1881 / 46,600$ & $4.04(3.86 ; 4.22)$ & Reference & $<0.0001$ \\
\hline 1 or + & $1143 / 15,018$ & $7.61(7.18 ; 8.07)$ & $1.77(1.64 ; 1.91)$ & \\
\hline Missing & $11 / 481$ & $2.29(1.14 ; 4.09)$ & & \\
\hline \multicolumn{5}{|l|}{ Educational level } \\
\hline$<$ High-school degree & 275/7178 & $3.83(3.39 ; 4.31)$ & Reference & \\
\hline High-school degree or undergraduate & $1311 / 26,739$ & $4.90(4.64 ; 5.18)$ & $1.35(1.19 ; 1.54)$ & $<0.0001$ \\
\hline Graduate degree or doctorate & $1147 / 22,155$ & $5.18(4.88 ; 5.49)$ & $1.56(1.37 ; 1.78)$ & $<0.0001$ \\
\hline Missing & $302 / 6027$ & $5.01(4.46 ; 5.61)$ & & \\
\hline \multicolumn{5}{|l|}{ Professional activity before lockdown } \\
\hline Student & $36 / 446$ & $8.07(5.65 ; 11.2)$ & $1.18(0.85 ; 1.64)$ & 0.3221 \\
\hline Working & $2025 / 30,170$ & $6.71(6.42 ; 7.01)$ & Reference & \\
\hline Looking for a job & $113 / 1578$ & $7.16(5.90 ; 8.61)$ & $1.01(0.84 ; 1.22)$ & 0.8955 \\
\hline Retired & $740 / 27,739$ & $2.67(2.48 ; 2.87)$ & $0.43(0.39 ; 0.47)$ & $<0.0001$ \\
\hline Not working due to health conditions & $48 / 529$ & $9.07(6.69 ; 12.0)$ & $1.35(1.01 ; 1.79)$ & 0.0415 \\
\hline No professional activity (housewife or husband) & $62 / 1490$ & $4.16(3.19 ; 5.33)$ & $0.64(0.50 ; 0.83)$ & 0.0006 \\
\hline Missing & $11 / 147$ & $7.50(3.74 ; 13.4)$ & & \\
\hline
\end{tabular}

Essential job position 
Table 2 Unweighted incidence rates of COVID-19-Like Symptoms (CLS) by covariate values and univariable hazard-ratios ${ }^{a}$ (Continued)

\begin{tabular}{|c|c|c|c|c|}
\hline & $\begin{array}{l}\text { Number CLS } \\
\text { /number person-months }\end{array}$ & $\begin{array}{l}\text { Incidence rate }(95 \% \mathrm{Cl}) \\
\text { (/100 person-months) }\end{array}$ & Hazard-Ratio & $P$-Value \\
\hline Healthcare worker (Y vs N) & $161 / 2207$ & $7.29(6.21 ; 8.51)$ & $1.44(1.23 ; 1.69)$ & $<0.0001$ \\
\hline Other essential job (Y vs N) & $361 / 5750$ & $6.28(5.65 ; 6.96)$ & $1.20(1.08 ; 1.35)$ & 0.0010 \\
\hline \multicolumn{5}{|l|}{ Professional activity during lockdown } \\
\hline Not working & $999 / 31,782$ & $3.14(2.95 ; 3.34)$ & $0.56(0.51 ; 0.62)$ & $<0.0001$ \\
\hline Stopped working & $173 / 2321$ & $7.45(6.38 ; 8.65)$ & $1.12(0.95 ; 1.32)$ & 0.1691 \\
\hline Working from home, remote working & $959 / 15,052$ & $6.37(5.97 ; 6.79)$ & Reference & \\
\hline Partially working from home & $188 / 3143$ & $5.98(5.16 ; 6.90)$ & $0.97(0.83 ; 1.14)$ & 0.7257 \\
\hline Working outside home & $313 / 4573$ & $6.84(6.11 ; 7.65)$ & $1.12(0.99 ; 1.27)$ & 0.0835 \\
\hline Other & $68 / 951$ & $7.15(5.55 ; 9.07)$ & $1.07(0.83 ; 1.36)$ & 0.6136 \\
\hline Missing & $335 / 4277$ & $7.83(7.02 ; 8.72)$ & & \\
\hline \multicolumn{5}{|l|}{ BMI $\left(\mathrm{kg} / \mathrm{m}^{2}\right)$} \\
\hline$<18.5$ & $104 / 2035$ & $5.11(4.18 ; 6.19)$ & $1.15(0.94 ; 1.40)$ & 0.1746 \\
\hline$[18.5 ; 25[$ & $1621 / 35,552$ & $4.56(4.34 ; 4.79)$ & Reference & \\
\hline [25; 30[(overweight) & $826 / 16,650$ & $4.96(4.63 ; 5.31)$ & $1.08(0.99 ; 1.17)$ & 0.0763 \\
\hline$>=30$ (obese) & $365 / 5615$ & $6.50(5.85 ; 7.20)$ & $1.40(1.25 ; 1.56)$ & $<0.0001$ \\
\hline Missing & $119 / 2247$ & $5.30(4.39 ; 6.34)$ & & \\
\hline \multicolumn{5}{|l|}{ Chronic diseases } \\
\hline Yes & $1005 / 20,670$ & $4.86(4.57 ; 5.17)$ & $1.12(1.03 ; 1.21)$ & 0.0053 \\
\hline No & $1993 / 40,834$ & $4.88(4.67 ; 5.10)$ & Reference & \\
\hline Didn't know & $34 / 461$ & $7.38(5.11 ; 10.3)$ & $1.73(1.23 ; 2.43)$ & 0.0015 \\
\hline Missing & $3 / 135$ & $2.23(0.46 ; 6.51)$ & & \\
\hline \multicolumn{5}{|l|}{ Chronic diseases (Y vs N) } \\
\hline Asthma, COPD, other resp. diseases & $415 / 8319$ & $4.99(4.52 ; 5.49)$ & $1.51(1.35 ; 1.69)$ & $<0.0001$ \\
\hline Diabetes & $113 / 3582$ & $3.15(2.60 ; 3.79)$ & $1.00(0.83 ; 1.22)$ & 0.9662 \\
\hline Hypertension & $270 / 6780$ & $3.98(3.52 ; 4.49)$ & $0.92(0.81 ; 1.04)$ & 0.1682 \\
\hline Other cardiovascular diseases & $79 / 2194$ & $3.60(2.85 ; 4.49)$ & $0.88(0.70 ; 1.10)$ & 0.2493 \\
\hline Cancer & $168 / 3286$ & $5.11(4.37 ; 5.95)$ & $1.14(0.97 ; 1.34)$ & 0.1078 \\
\hline Anxiety, depression & $132 / 1702$ & $7.75(6.49 ; 9.20)$ & $1.72(1.44 ; 2.04)$ & $<0.0001$ \\
\hline Other & $432 / 8003$ & $5.40(4.90 ; 5.93)$ & $1.27(1.15 ; 1.41)$ & $<0.0001$ \\
\hline Missing & $3 / 135$ & $2.23(0.46 ; 6.51)$ & & \\
\hline \multicolumn{5}{|c|}{ Positive RT-PCR in another household member } \\
\hline$(Y$ vs $N)$ & $58 / 207$ & $28.0(21.2 ; 36.1)$ & $5.68(4.38 ; 7.37)$ & $<0.0001$ \\
\hline
\end{tabular}

${ }^{a}$ with stratification on cohort study

recommendations. This low proportion can be explained in part by the need for some participant to go out for basic necessities, for example if the participant lived alone.

Considering the estimated 5-day median incubation time of COVID-19 and the appearance of symptoms within twelve days after infection [14], a large proportion of participants who developed CLS in the first two weeks were probably infected before lockdown, most of them in the community or at the workplace. It is therefore not surprising to find the association of CLS in adults with decreasing age [15], living in urban versus rural environments [16], in highly prevalent French regions [17], all factors that were reported in other studies performed before lockdown.

A lower infection rate with increasing age was reported in several population-based serological studies [18] which is consistent with our findings, although the risk of severe illness or deaths exponentially increases with age among those infected [19]. As in other studies, univariable analysis identified the size of the household (including children), but only living with at least one 
Table 3 Multivariable-adjusted hazard-ratios of COVID-19-Like Symptoms (CLS) according to covariate values ${ }^{\text {a }}$

\begin{tabular}{|c|c|c|}
\hline & Hazard-Ratio & $P$-Value \\
\hline \multicolumn{3}{|l|}{ Age group } \\
\hline$<40$ & Reference & \\
\hline$[40-50[$ & $0.80(0.72 ; 0.90)$ & $<0.0001$ \\
\hline$[50-60[$ & $0.68(0.60 ; 0.76)$ & $<0.0001$ \\
\hline$[60-70[$ & $0.52(0.44 ; 0.62)$ & $<0.0001$ \\
\hline$>=70$ & $0.34(0.27 ; 0.44)$ & $<0.0001$ \\
\hline \multicolumn{3}{|l|}{ Gender } \\
\hline Female & reference & \\
\hline Male & $0.99(0.91 ; 1.07)$ & 0.7267 \\
\hline \multicolumn{3}{|l|}{ Regions } \\
\hline Ile-de-France & $1.31(1.19 ; 1.44)$ & $<0.0001$ \\
\hline GrandEst & $1.29(1.14 ; 1.47)$ & $<0.0001$ \\
\hline Other French metropolitan regions & reference & \\
\hline \multicolumn{3}{|l|}{ Living Area } \\
\hline Rural & Reference & \\
\hline$<20,000$ inhab. & $1.07(0.93 ; 1.22)$ & 0.3629 \\
\hline 20-000-100,000 inhab. & $1.14(1.00 ; 1.30)$ & 0.0471 \\
\hline > 100,000 inhab. & $1.15(1.02 ; 1.29)$ & 0.0241 \\
\hline \multicolumn{3}{|l|}{ Household size and composition } \\
\hline \multicolumn{3}{|l|}{ Nb persons (incl. participant) } \\
\hline 1 & Reference & \\
\hline 2 & $0.95(0.85 ; 1.06)$ & 0.3405 \\
\hline 3 or + & $1.00(0.87 ; 1.16)$ & 0.9603 \\
\hline \multicolumn{3}{|l|}{$\mathrm{Nb}$ children (< $18 \mathrm{yrs})$} \\
\hline 0 & Reference & \\
\hline 1 or + & $1.28(1.13 ; 1.45)$ & 0.0001 \\
\hline \multicolumn{3}{|l|}{ BMI (kg/m2) } \\
\hline$<18.5$ & $1.04(0.85 ; 1.27)$ & 0.6916 \\
\hline$[18.5 ; 25[$ & Reference & \\
\hline [25; 30[(overweight) & $1.17(1.07 ; 1.27)$ & 0.0005 \\
\hline$>=30$ (obese) & $1.41(1.25 ; 1.58)$ & $<0.0001$ \\
\hline \multicolumn{3}{|l|}{ Professional activity before lockdown } \\
\hline Student & $1.01(0.71 ; 1.44)$ & 0.9620 \\
\hline Working & Reference & \\
\hline Looking for a job & $1.05(0.86 ; 1.27)$ & 0.6618 \\
\hline Retired & $0.86(0.72 ; 1.03)$ & 0.1057 \\
\hline Not working due to health conditions & $1.30(0.96 ; 1.77)$ & 0.0894 \\
\hline No professional activity (house wife or husband) & $0.89(0.68 ; 1.16)$ & 0.3773 \\
\hline \multicolumn{3}{|l|}{ Essential job position } \\
\hline Health care worker (Y vs N) & $1.18(1.00 ; 1.40)$ & 0.0535 \\
\hline Other essential job (Y vs N) & $1.01(0.90 ; 1.14)$ & 0.8922 \\
\hline \multicolumn{3}{|l|}{ Chronic diseases (Y vs N) } \\
\hline Asthma, COPD, other resp. diseases & $1.41(1.24 ; 1.60)$ & $<0.0001$ \\
\hline Anxiety, depression & $1.31(1.08 ; 1.58)$ & 0.0065 \\
\hline
\end{tabular}


Table 3 Multivariable-adjusted hazard-ratios of COVID-19-Like Symptoms (CLS) according to covariate values ${ }^{\mathrm{a}}$ (Continued)

\begin{tabular}{ll}
\hline Other & Hazard-Ratio \\
\hline a with stratification on the cohort. $6567(6 \%)$ participants excluded from the multivariable model due to missing values including 190 with CLS; educational level \\
was not significantly associated with CLS when included in the multivariable model (high school degree or undergraduate versus $<$ high-school degree, $P=0.7743 ;$ \\
graduate or doctorate versus $<$ high-school degree, $P=0.6299)$, and was not kept in the final model due to missing information on this covariate in the E4NG2 \\
cohort. To avoid overfitting Positive RT-PCR in another household member was not entered in the model. However, results were unchanged when this variable \\
was entered (not shown)
\end{tabular}

child or adolescent remained associated with CLS on multivariable analysis, indicating that this age group could play an important role in household-related transmission [20]. We also identified other factors indicating potential secondary household-related transmission, such as living with another person with a positive diagnosis of SARS-CoV-2 [21]. However, it was impossible to determine a timeframe for this factor and identify whether the participant was the source of infection or was infected by a household member. Being a healthcare worker was associated with CLS in univariable analysis as reported other studies [22, 23], but the association did not remain significant in the multivariable model, potentially due to a lack of statistical power. Obesity has been found to be linked with the risk of severe CLS in young patients [24], and also suspected to increase the susceptibility to infection [25]. Different theories suggest that asthma, COPD and other respiratory diseases may be negatively or positively associated with the susceptibility to SARS-CoV-2 infection due to up or down regulation of angiotensin-converting enzyme- 2 expression. However, all of these respiratory diseases have been shown to be associated with the severity of illness in infected persons [26-28]. Since 30 to $60 \%$ of SARS-CoV-2 infections are asymptomatic [29-32] and were not included in our CLS cases, by definition, it is not surprising to find the presence of these conditions, which are known to be associated with more severe disease, in subjects with symptomatic SARS-CoV-2 infection. Finally, we found a strong association between CLS and anxiety or depression, which may be related either to a direct (i.e. causal) impact of these comorbidities on the risk of CLS, or to an over-reporting of CLS caused by increased anxiety or stress in this specific subgroup. Although psychiatric disorders have been reported during the acute phase of the infection [33], the risk of reverse causality explaining this association should however be limited as co-morbidities were collected prior to the survey.

Consistent results were obtained in the sensitivity analyses. An association with CLS was found with being retired compared with being working, but the strength of the association was of the same magnitude than estimated in the main analysis. This result can be the consequence of a higher power of the sensitivity analyses due to a higher number of events, and explained by a lower rate of social contacts in this category of persons compared with working people of the same age.

Our study has several limitations. The most important limitation is the lack of virological confirmation of CLS and the risk of misclassification of a SARS-CoV-2 infection and a disease from another etiology. During lockdown, French health authority recommendations limited SARS-CoV-2 testing with a RT-PCR test to patients with severe symptoms requiring hospitalization or to specific situations (e.g. healthcare workers with symptoms). Thus, testing was not available to most participants. Nevertheless, the influenza season peaked on week 6 and ended on week 10 to 12, just before lockdown, which limits the risk of acute respiratory infection caused by an influenza virus. In addition, $42 \%$ of the small group of participants who were tested for SARSCoV-2 infection in our study reported a positive RTPCR result. This positive rate was higher than that reported in the community $(30 \%$ at its highest between March 23 and March 29, 2020) [34]. However, a 15 to $20 \%$ seroprevalence of SARS-CoV-2 was reported in Spain in individuals from the general population who presented symptoms compatible with COVID-19 [32]. It is therefore likely that the cause of illness was not SARS$\mathrm{CoV}-2$ infection in a significant proportion of our CLS cases and only studies using sensitive and specific virological methods can accurately quantify the extent of the SARS-CoV-2 epidemic. To avoid recall bias, which is another potential limitation of our study, we limited the questionnaire to the symptoms present in the past 14 days. To avoid a selection bias induced by different dates for filling in the questionnaires resulting in dates of 'atrisk period' that varied from one subject to another, we used a Cox model with delayed entry. Finally, although participation bias was accounted for with an appropriate weighting method, our findings should not be considered to be strictly representative of the general adult population in France. Nevertheless, the large number of subjects from all social categories allows us to draw robust conclusions on the factors associated with the occurrence of CLS in France.

\section{Conclusion}

In conclusion, to our knowledge this is the first study to quantify the incidence of CLS in the general population on a nationwide scale and during a lockdown, and it 
shows the significant impact of lockdown on the dynamics of the incidence of infection. A follow-up study is ongoing and will be combined with SARS-CoV-2 serological tests of all participants to estimate the seroprevalence and identify the associated factors.

\section{Supplementary Information}

The online version contains supplementary material available at https://doi. org/10.1186/s12879-021-05864-8

\section{Additional file 1.}

\section{Abbreviations}

OR: Odds-Ratio: Cl: Confidence Intervals

\section{Acknowledgments}

The authors warmly thank all the volunteers of the CONSTANCES, E3N-E4N, and NutriNet-Santé cohorts.

We thank the staff of the CONSTANCES, E3N-E4N and NutriNet-Santé cohorts that have worked with dedication and engagement to collect and manage the data used for this study and to ensure continuing communication with the cohort participants.

We thank Mrs. Dale Roche-Leclerc for her help in editing the manuscript. The SAPRIS study group.

Nathalie Bajos (co-Principal investigator), Fabrice Carrat (co-Principal investigator), Pierre-Yves Ancel, Marie-Aline Charles, Florence Jusot, Claude Martin, Laurence Meyer, Ariane Pailhé, Gianluca Severi, Alexis Spire, Mathilde Touvier, Marie Zins.

\section{Authors' contributions}

Study idea and design: Carrat, Touvier, Severi, Meyer, Jusot, Charles, Ancel, Zins, Bajos. Data acquisition: Touvier, Severi, Zins. Data analysis and interpretation: Carrat, Touvier, Severi, Meyer, Jusot, Zins, Bajos. Drafting of the manuscript: Carrat. Critical revision of the manuscript for important intellectual content: All authors. Statistical analysis: Carrat, Lapidus. Obtained funding: Carrat, Bajos. Administrative, technical, or material support: Rahib, Lydié. Study supervision: Carrat, Touvier, Severi, Charles, Ancel, de Lamballerie, Zins, Bajos. The author (s) read and approved the final manuscript.

\section{Funding}

Role of the funding source.

Sponsor and funding sources played no role in the study design, data collection, analysis, interpretation or drafting of the study. FC had full access to all data in the study and FC and NB made the final decision to submit the study for publication.

This study.

ANR (Agence Nationale de la Recherche, \#ANR-20-COVI-000,\#ANR-10-COHO06), Fondation pour la Recherche Médicale (\#20RR052-00), Inserm (Institut National de la Santé et de la Recherche Médicale, \#(20-26).

Cohorts funding.

The CONSTANCES Cohort Study is supported by the Caisse Nationale d'Assurance Maladie (CNAM), the French Ministry of Health, the Ministry of Research, the Institut national de la santé et de la recherche médicale. CONSTANCES benefits from a grant from the French National Research Agency [grant number ANR-11-INBS-0002] and is also partly funded by MSD, AstraZeneca, Lundbeck and L'Oreal.

The E3N-E4N cohort is supported by the following institutions: Ministère de I'Enseignement Supérieur, de la Recherche et de I'Innovation, INSERM, University Paris-Saclay, Gustave Roussy, the MGEN, and the French League Against Cancer.

The NutriNet-Sante study is supported by the following public institutions: Ministère de la Santé, Santé Publique France, Institut National de la Santé et de la Recherche Médicale (INSERM), Institut National de la Recherche Agronomique (INRAE), Conservatoire National des Arts et Métiers (CNAM) and Sorbonne Paris Nord.

\section{Availability of data and materials}

In regards to data availability, data of the study are protected under the protection of health data regulation set by the French National Commission on Informatics and Liberty (Commission Nationale de l'Informatique et des Libertés, CNIL). The data can be available upon reasonable request to the corresponding author (fabrice.carrat@iplesp.upmc.fr), after a consultation with the steering committee of the Sapris study. The French law forbids us to provide free access to Sapris data; access could however be given by the steering committee after legal verification of the use of the data.

\section{Ethics approval and consent to participate}

Ethical approval and written informed consent was obtained from each participant before enrolment in the original cohort. The study was approved by the Inserm ethics evaluation committee (approval \#20-672 dated March 30, 2020). According to French law, the present nested survey did not require specific additional written consent from the participant. Representatives of the participants tested and validated the questionnaires, but they did not contribute to other aspects related to the design, conduct, reporting or dissemination of the research.

\section{Consent for publication}

NA

\section{Competing interests}

Prof Fabrice Carrat, Mathilde Touvier, Prof Gianluca Severi, Prof Laurence Meyer, Prof Florence Jusot, Nathanael Lapidus, Delphine Rahib, Nathalie Lydié, Marie-Aline Charles, Prof Pierre-Yves Ancel, Alexandra Rouquette,

Claude Martin, Prof Xavier de Lamballerie, Prof Marie Zins, Nathalie Bajos declare no competing interest.

\section{Author details}

${ }^{1}$ Sorbonne Université, Inserm, Institut Pierre-Louis d'Epidémiologie et de Santé Publique, 27 rue Chaligny, 75571 CEDEX 12 Paris, France.

2Département de Santé Publique, APHP. Sorbonne Université, Paris, France. ${ }^{3}$ Sorbonne Paris Nord University, Inserm U1153, Inrae U1125, Cnam, Nutritional Epidemiology Research Team (EREN), Epidemiology and Statistics Research Center - University of Paris (CRESS), Bobigny, France. ${ }^{4}$ CESP UMR1018, Université Paris-Saclay, UVSQ, Inserm, Gustave Roussy, Villejuif, Paris, France. ${ }^{5}$ Department of Statistics, Computer Science and Applications, University of Florence, Florence, Italy. ${ }^{6}$ Université Paris Saclay, Inserm, CESP U1018, Le Kremlin Bicêtre, Paris, France. ${ }^{7}$ Service de Santé Publique, APHP. Paris Saclay, Le Kremlin Bicêtre, France. ${ }^{8}$ Université Paris-Dauphine, PSL-Research University, LEDa, Paris, France. ${ }^{9}$ Santé publique France, Saint-Maurice, France. ${ }^{10}$ Ined, Inserm, EFS, UMS Elfe, Aubervilliers, Paris, France. ${ }^{11}$ Obstetrical, Perinatal and Pediatric Epidemiology Research Team, Center for Epidemiology and Statistics Sorbonne Paris Cité, INSERM U1153, Paris Descartes University, Paris, France. ${ }^{12}$ Clinical Research Unit, Center for Clinical Investigation P1419, Cochin Broca Hôtel-Dieu Hospital, Paris, France.

${ }^{13}$ Unité des Virus Emergents, UVE: Aix Marseille Univ, IRD 190, INSERM 1207, IHU Méditerranée Infection, 13005 Marseille, France. ${ }^{14}$ Paris University, Paris, France. ${ }^{15}$ Paris Saclay University, Inserm UMS 11, Villejuif, France. ${ }^{16}$ IRIS, Inserm/EHESS/CNRS, Aubervilliers, France.

Received: 7 October 2020 Accepted: 21 January 2021

Published online: 10 February 2021

\section{References}

1. Li Q, Guan X, Wu P, et al. Early transmission dynamics in Wuhan, China, of novel coronavirus-infected pneumonia. N Engl J Med. 2020;382(13):1199-207.

2. Bernard Stoecklin S, Rolland $P$, Silue $Y$, et al. First cases of coronavirus disease 2019 (COVID-19) in France: surveillance, investigations and control measures, January 2020. Euro Surveill. 2020;25(6):2000094.

3. Info Coronavirus COVID-19 - Stratégie de déconfinement. Gouvernement.fr. 2020. https://www.gouvernement.fr/info-coronavirus/strategie-dedeconfinement. Accessed 4 May 2020..

4. Flaxman S, Mishra S, Gandy A, et al. Estimating the effects of nonpharmaceutical interventions on COVID-19 in Europe. Nature. 2020; 584(7820):257-61.

5. Nombre quotidien de personnes nouvellement hospitalisées pour covid-19. https://geodes.santepubliquefrance.fr/\#c=indicator\&i=covid hospit incid. incid_hosp\&s=2020-07-09\&t=a01\&view=map 1 (accessed July 10, 2020). 2020 
6. Cauchemez S, Kiem CT, Paireau J, Rolland P, Fontanet A. Lockdown impact on COVID-19 epidemics in regions across metropolitan France. Lancet. 2020; 396(10257):1068-9.

7. Salje H, Tran Kiem C, Lefrancq N, et al. Estimating the burden of SARS-CoV-2 in France. Science. 2020;369(6500):208-11.

8. Zins M, Goldberg M, Team C. The French CONSTANCES population-based cohort: design, inclusion and follow-up. Eur J Epidemiol. 2015;30(12):1317-28.

9. Clavel-Chapelon F, Group ENS. Cohort profile: the French E3N cohort study. Int J Epidemiol. 2015;44(3):801-9.

10. Hercberg S, Castetbon K, Czernichow S, et al. The Nutrinet-Sante study: a web-based prospective study on the relationship between nutrition and health and determinants of dietary patterns and nutritional status. BMC Public Health. 2010;10:242.

11. European Center for Diseases Control. Case definition for coronavirus disease 2019 (COVID-19), as of 29 May 2020. https://www.ecdc.europa.eu/ en/covid-19/surveillance/case-definition (accessed June 15th 2020).

12. WHO. Consultation on Obesity. Obesity: preventing and managing the global epidemic: report of a WHO consultation (WHO technical report series; 894). Geneva: WHO; 1999.

13. Pullano G, Di Domenico L, Sabbatini CE, et al. Underdetection of COVID-19 cases in France threatens epidemic control. Nature. 2020;(Dec 21). https:// doi.org/10.1038/s41586-020-03095-6.

14. Lauer SA, Grantz KH, Bi Q, et al. The incubation period of coronavirus disease 2019 (COVID-19) from publicly reported confirmed cases: estimation and application. Ann Intern Med. 2020;172(9):577-82

15. Lapointe-Shaw L, Rader B, Astley CM, et al. Web and phone-based COVID19Syndromic surveillance in Canada: a cross-sectional study. PLoS One. 2020;15(10):e0239886.

16. Rader B, Nande A, Adlam B, et al. Crowding and the shape of COVID-19 epidemics. Nat Med. 2020;(Oct 5). https://doi.org/10.1038/s41591-020-1104-0.

17. Sante-Publique-France. COVID-19 Point épidémiologique - Situation au 15 mars 2020 à minuit. 2020. https://www.santepubliquefrance.fr/maladies-ettraumatismes/maladies-et-infections-respiratoires/infection-a-coronavirus/ documents/bulletin-national/covid-19-point-epidemiologique-du-15-mars-2 020 (accessed June 25, 2020).

18. Bobrovitz N, Arora RK, Cao C, et al. Global seroprevalence of SARS-SoV-2 antibodies: a systematic review and meta-analysis. medRxiv 2020; preprint November 18, 2020: doi: https:/doi.org/https:/doi.org/10.1101/2020.11.17.20233460.

19. O'Driscoll M, Ribeiro Dos Santos G, Wang L, et al. Age-specific mortality and immunity patterns of SARS-CoV-2. Nature. 2020;(Nov 2). https://doi.org/10.1 038/s41586-020-2918-0.

20. Jing QL, Liu MJ, Zhang ZB, et al. Household secondary attack rate of COVID19 and associated determinants in Guangzhou, China: a retrospective cohort study. Lancet Infect Dis. 2020;(Jun 17) S1473-3099(20)30471-0. https://doi.org/10.1016/S1473-3099(20)30471-0.

21. Lei H, Xu X, Xiao S, Wu X, Shu Y. Household transmission of COVID-19-a systematic review and meta-analysis. J Inf Secur. 2020;81 (6):979-97.

22. Zhan M, Qin Y, Xue X, Zhu S. Death from Covid-19 of 23 health Care Workers in China. N Engl J Med. 2020;382(23):2267-8.

23. Gomez-Ochoa SA, Franco OH, Rojas LZ, et al. COVID-19 in health-care workers: a living systematic review and meta-analysis of prevalence, risk factors, clinical characteristics, and outcomes. Am J Epidemiol. 2021;190(1):161-75.

24. Ong SWX, Young BE, Leo YS, Lye DC. Association of higher body mass index (BMI) with severe coronavirus disease 2019 (COVID-19) in younger patients. Clin Infect Dis. 2020. 2020 May 8:ciaa548. https:/doi.org/10.1093/cid/ciaa548.

25. Belancic A, Kresovic A, Racki V. Potential pathophysiological mechanisms leading to increased COVID-19 susceptibility and severity in obesity. Obes Med. 2020;19:100259.

26. Halpin DMG, Faner $R$, Sibila O, Badia JR, Agusti A. Do chronic respiratory diseases or their treatment affect the risk of SARS-CoV-2 infection? Lancet Respir Med. 2020;8(5):436-8.

27. Leung JM, Yang CX, Tam A, et al. ACE-2 expression in the small airway epithelia of smokers and COPD patients: implications for COVID-19. Eur Respir J. 2020;55(5):2000688.

28. Zhao Q, Meng M, Kumar R, et al. The impact of COPD and smoking history on the severity of COVID-19: A systemic review and meta-analysis. J Med Virol. 2020;(Apr 15). https://doi.org/10.1002/jmv.25889.

29. Kimball A, Hatfield KM, Arons M, et al. Asymptomatic and Presymptomatic SARS-CoV-2 infections in residents of a long-term care skilled nursing facility - King County, Washington, march 2020. MMWR Morb Mortal Wkly Rep. 2020;69(13):377-81.
30. Nishiura $H$, Kobayashi T, Miyama $T$, et al. Estimation of the asymptomatic ratio of novel coronavirus infections (COVID-19). Int J Infect Dis. 2020:94:154-5.

31. Oran DP, Topol EJ. Prevalence of asymptomatic SARS-CoV-2 infection: a narrative review. Ann Intern Med. 2020;173(5):362-7.

32. Pollan M, Perez-Gomez B, Pastor-Barriuso R, et al. Prevalence of SARS-CoV-2 in Spain (ENE-COVID): a nationwide, population-based seroepidemiological study. Lancet. 2020;396(10250):535-44.

33. Rogers JP, Chesney E, Oliver D, et al. Psychiatric and neuropsychiatric presentations associated with severe coronavirus infections: a systematic review and meta-analysis with comparison to the COVID-19 pandemic. Lancet Psychiatry. 2020;7(7):611-27.

34. Sante-Publique-France. COVID-19 Point épidémiologique hebdomadaire du 25 juin 2020. 2020. https://www.santepubliquefrance.fr/maladies-et-trauma tismes/maladies-et-infections-respiratoires/infection-a-coronavirus/ documents/bulletin-national/covid-19-point-epidemiologique-du-25-juin-2 020 (accessed June 27, 2020 2020)

\section{Publisher's Note}

Springer Nature remains neutral with regard to jurisdictional claims in published maps and institutional affiliations.
Ready to submit your research? Choose BMC and benefit from:

- fast, convenient online submission

- thorough peer review by experienced researchers in your field

- rapid publication on acceptance

- support for research data, including large and complex data types

- gold Open Access which fosters wider collaboration and increased citations

- maximum visibility for your research: over $100 \mathrm{M}$ website views per year

At $\mathrm{BMC}$, research is always in progress.

Learn more biomedcentral.com/submissions 